\title{
Patient Satisfaction in Elective Major Surgery for Benign Disease in a Tertiary Care Hospital.
}

\author{
Shanta Bir Maharjan' ${ }^{1}$, Deep Lamichhane ${ }^{1}$, Arbin Joshi ${ }^{1}$, Sashi Shah', Ghanshyam Thapa ${ }^{1}$, Jay N Shah ${ }^{1}$. \\ ${ }^{1}$ Department of Surgery, Patan Hospital, Patan Academy of Health Science (PAHS).
}

\begin{abstract}
Introduction: Since civilization physicians are upheld at high standard to promote interests of patients and society. In this study we aimed to analyze issues related to patient satisfaction for better patient care.

Methods: There were 145 surgical patients in this cross sectional prospective observational study for elective benign disease. Patient's response to pre-tested questionnaire before and after surgery was scored was on a 3-point scale. Variables included patient demographics; diagnosis, treatments, complications; counseling, social, financial concern; surgeon's competency, hospital facilities, pain management and overall satisfaction. Microsoft Excel was used for descriptive analysis.
\end{abstract}

Results: Average age of patients was 44 years and male were $42.7 \%$ (62/145). All patients were satisfied for information on diagnosis, complications and hospital facilities. Patients consented for surgery themselves in $96.5 \%$. Financial concern was found in $17.3 \%$ and inadequate pain management in $7.6 \%$. Overall,96.5\% patients were satisfied.

Conclusions: Even though $96.5 \%$ patients were satisfied, further analysis revealed there is room for improvement on post-operative pain management (in $17.3 \%$ ) and financial constrains (in $7.6 \%$ ) in elective major surgery for benign diseases in our setup.

Keywords: consent, counseling, elective surgery, patients satisfaction.

\section{INTRODUCTION}

Since early civilization society has upheld physicians to a high standard to promote interests of patients and society. We need to make sure patients understand and willingly accept our services. The value of detail, valid, informed and understood consent before surgery is important for patient satisfaction and legal issues. ${ }^{1,2,3}$

Patients are often confused and vulnerable when sick. This is further complicated by lack of consistency in institutional, ethical and legal policies. ${ }^{4}$ Patients with serious illness like cancer may leave the worry of technicality and difficult choices to surgeon. ${ }^{5}$ In milder illness like hernia repair patients are relatively at ease and may demand exhausting details. In clinical practice we encounter wide spectrum of patient reaction from distrust to full faith in surgeon. ${ }^{6}$

Despite complexities, surgeons and patients manage doubt and negotiate together to achieve success. Failing in this duty, may result in un-satisfaction, frustration and litigation. 'Counseling and informed consent' has evolved as major part of our dutyin today's medicine. In this study we aim to document 'specific details' of the process which is often poor and topic of debate. Spending 'adequate' time to explain before procedure is important especially when things do not go as planned. In this study we aimed

\section{Correspondence:}

Prof. Dr. Jay N Shah

Department of Surgery, Patan Academy of Health Sciences (PAHS)

Lalitpur, Kathmandu, Nepal.

Email: drjaywufei@gmail.com

Phone: 977-9851040139 
to document issues related to patient satisfaction in order to plan for further intervention.

\section{METHODS}

This was a cross sectional prospective observational study from Feb to April 2011 with aim of enrolling 150 cases. Adult patients scheduled for elective benign major surgery were included. Patients with malignant disease or during emergency surgery were excluded because they have different psychology and demands. Ethical approval was obtained from institutional review committee. Pre determinedproforma (modified as per earlier pilot study in 20 consecutive cases) was used to collect data. Questions were divided in two parts. Variables like gender, age, procedure and patients' understanding of diagnosis, treatment were documented during counseling in outpatient department. Patients were encouraged to sign'written informed understood consent form' (WIUCF) by themselves. Some questions were asked after surgery in the ward before discharge in order to decrease patient's anxiety and avoid biasness, like how patient felt of the surgeon's competency or hospital facilities. (Table 1).

After discussion, patients understanding and satisfaction was scored as per set criteria- if patient could recall at least $50 \%$ of information on complications (general 3, specific 1), then it was considered satisfactory (score 2). General complications included those which are common to all surgeries- anesthesia complication, drug reaction, wound infection, bleeding, re-surgery, persisting symptom etc. Specific complications included exclusive to particular surgery- for example cholecystectomy for gallstones- bile duct injury, bile leak, retained stone in common bile duct, recurrent bile duct stone etc. For '\%' of complications, patients were not required to recall value (e.g. $0.2-0.6 \%$ chance of bile duct injury during cholecystectomy). If patient could recall that they were told about ' $\%$ ' then it was considered satisfactory (score 2). Alternative treatment offered means patients were told about possible non-surgical management including their pros and cons, e.g. medical management for benign enlargement of prostate.

All patients were seen and counseled by consultant in outpatient department before putting in operation list. Detail counseling and documentation was done by resident or registrar familiar with the results of pilot study. Postoperative information on first day after surgery was obtained by different resident who was not involved in pre operative documentation to reduce biasness.

Three point score with 'yes 2, not sure 1, no 0' were given for each variable. Microsoft Excel was used for data analysis.

\section{RESULTS}

There were 145 patients during study period. Average age was 44 years (range 14 to 73 ). Male were $42.7 \%$ (62/145). Overall 140 (96.5\%) patients were satisfied. All patients were satisfied for information provided on nature of illness (name/diagnosis), alternative treatment and complications. Patients gave consent themselves for surgery in $96.5 \%(140 / 145)$ cases. Concerns for return to job and financial issues were found in $17.3 \%$ (25/145). All patients expressed satisfaction with regard to the surgeons' competency, hospital facilities and discharge advice. Eleven patients (7.6\%) were not satisfied for nursing care and post-operative pain management.

Table 1. Patients $(n=145)$ response on their understanding and satisfaction for elective major surgery for benign disease.

\section{Following questions were asked pre-operatively in outpatient department during counseling and} consenting.

1. Have you been explained about followings? (score: yes $=2$, not sure $=1$, no $=0$ )

Name of disease/diagnosis, alternative treatment, necessity of surgery, type of surgery (major/ intermediate/minor), complications (name, \%, possible death).,

2. Do you have these issues to make you concerned about surgery?

Financial, family, leave from job, return to same job.

3. You will need to sign the WIUCF to authorize us to perform surgery.

Consent given by patient (score 2) / family (score 1) / other (score 0 ).

Following questions were asked on first post-operative day in the ward before discharge.

1. Do you have concern about? (score: yes $=2$, not sure=1, no=0)

Competency of surgeon, hospital facilities, nursing care, pain management, discharge advice (on diet, medication, follow-up).

2. How will you rate your overall satisfaction? (score: yes $=2$, not sure $=1$, no= 0 )

*WIUCF= written informed understood consent form

\section{DISCUSSION}

We had $17.3 \%$ (25/145) patients who had financial concern and were unsatisfied. This number is not as high as one would expect in our low income society where patients pay from their pocket. Possible explanation could be Patan Hospital is a 'not for profit' public institute with affordable expenses. The issue of finance and medical 
insurance is a complex issue. This is not solely in the domain of surgeons and individual institute. Society and state has responsibility to make health service affordably available to every citizen.

Research and appropriate measure is needed to look in to the issues of concern to patients who undergo major changes in life due to surgery. Such measures are necessary to satisfy patient, family and society as a whole. Satisfied patients are less likely to go push for litigation and lawsuits. In this study, we chose 3-point scale (instead of more reliable ' 5 or 10 point') for ease and consistency in scoring different variables. Patient satisfaction is an important indicator of the quality in health care. It affects clinical outcomes and medico-legal issues. ${ }^{7}$

We had $7.6 \%$ of patients who were not satisfied with pain management. This could be higher given the facts that patients in our society are expected to accept certain degree of pain. However, this is our duty to make our patients as pain free as possible.

At our institute supplies for treatment/surgery are provided by hospital and charged for at the time of discharge. Family members are not required to be on standby and run for the supplies as and when needed. This is a great relief to family and attendants not to be 'on toes' during or after surgery. Institution where surgery is performed together with competency, commitment is key factors in patient satisfaction. ${ }^{8}$ All patients in this study were satisfied with surgeons competency and hospital facilities. To minimize bias, we asked about competency and facilities on first day after surgery. We chose not wait to measure satisfaction during follow up in outpatient department because many patients do not turn up for regular follow up.

At our institute, we encourage competent adults to provide consent themselves. We had 96.5\% (140/145) of patients signed WIUCF themselves. WIUCF is a voluntary authorization given to surgeon by the patient to carry out surgery. This guides both patients and surgeons to exchange and document information on procedures, risks-benefits of having or not having the procedure and any alternative treatments with possible outcome. ${ }^{9,10}$ However, there is lack of agreement on how much information to include in WIUCF for different procedures in various subspecialties. ${ }^{11}$ Counseling and obtaining consent from patient 'themselves' is considered better for overall satisfaction when they are allowed to participate in the process. Research has shown that patients are more satisfied when involved in discussion process. Patients' involvement in decision making has increased over time, but not in every respect. ${ }^{12}$
In this study our aim was to involve patient in the process of decision making to improve their satisfaction. Overall $96.5 \%$ patients were satisfied. Varying consenting practice and failure to warn patients of significant complications can lead to unsatisfied patients. Structured consent form with details of significant risk and complication can improve consent process. ${ }^{13}$

In this study we initiated the process to improve surgical services by providing more information and getting patients involved in the process of decision making. Studies have shown that surgeon initiated efforts does improve safety and services in all specialties in all levels of hospitals. ${ }^{14}$

Possible limitation of this study could be lack of circumstances with 'complication or death' in the studied sample. In such conditions, patient and family may have different perception of overall satisfaction. Studies have shown that patients are less satisfied who develop complication. ${ }^{15}$ Future study can address the issues of satisfaction among patients with complications and/or death after surgery.

The new knowledge added by this study: Daily clinical surgical services at institution level needs improvement through periodical audit. Appropriate intervention is needed to increase patient satisfaction. For this, details of disease, diagnosis, treatment including alternatives and with outcome, nursing care especially post operative management and including financial issues of patients are key factors for the overall satisfaction.

Possible implications: The result of this study is useful in clinical practice for system improvement at institution level and evaluation of existing surgical services by addressing the patient satisfaction. Addressing post operative pain management and financial issues are important factors for overall patient satisfaction. Institutional social and national level program of medical insurance system is an option to address financial burden of the majority of patients. Periodical audit of the institutional services are necessary to evaluate and devise appropriate intervention.

\section{CONCLUSIONS}

Patient satisfaction is the key component of good surgical services. Overall we had $96.5 \%$ satisfied patients. This study points out need for improvement on post-operative pain management and financial constrains in elective major surgery for benign diseases in our setup. 


\section{REFERENCES}

1. Variations in consenting practice for laparoscopic Cholecystectomy. Ann R CollSurg Engl. 2006;88:482-5.

2. Informed consent in renal transplantation. Postgrad Med J. 2005;81:188-90.

3. Scarrow AM, Scarrow MR. Informed consent for the neurosurgeon. Surg Neurol. 2002;57:63-9.

4. Schneider CE. Editorial: some realism about informed consent. J Lab Clin Med. 2005;145:289-91.

5. McKneally MF, Martin DK. An entrustment model of consent for surgical treatment of lifethreatening illness: perspective of patients requiring esophagectomy. J Thorac Cardio-vasc Surg. 2000;120:264-9.

6. McKneally MF, Ignagni E, Martin DK et al. The leap to trust: perspective of cholecystectomy patients on informed deci-sion-making and consent. J Am Coll Surg. 2004;199:51-7.

7. BhanuPrakash. Patient Satisfaction.J CutanAesthet Surg. 2010;3 (3)151-5.

8. Responding to Trust: Surgeons' Perspective on Informed Consent. World J Surg. 2009;33:1341-7.

9. Feld AD: Informed consent: not just for procedures anymore. Am J Gastroenterol.2004;99 (6):977-80.

10. Yoshiyuki Takimoto, Shoichi Maeda, Brian
Taylor Slingsby, Kenji Harada, Takahide Nagase, HirokazuNagawa, Ryozo Nagai, Akira Akabayashi. A template for informed consent forms in medical examination and treatment: An intervention study. Med SciMonit. 2007;13 (8):PH15-8.

11. McManus PL, Wheatley KE. Consent and complications: risk disclosure varies widely between individual surgeons. Ann R CollSurg Engl. 2003;85:7982.

12. van den Brink-Muinen $A$, van Dulmen SM, de Haes HC, Visser AP, Schellevis FG, Bensing JM. Has patients' involvement in the decision-making process changed over time? Health Expect. 2006 Dec;9 (4):333-42.

13. Uzzaman MM, Tayeh S, Sinha S, Ratnasingham K, Stoker DL. Consentingpractice for laparoscopic cholecystectomy - are we doing enough to warn patients about their operation? Int J Surg. 2011;9 (8):643-7.

14. Hiram C. Polk Jr, Margaret B. Tyson, Susan Galandiuk. A situational overview of surgical quality and safety in 2010.Presented at the Symposium on Safety and Quality Improvement in Specialty Surgery in Smaller Hospitals, October 20, 2009, Louisville, KY.The American Journal of Surgery. 2010;200 (1): 82-89.

15. Colakoglu S, Khansa I, Curtis MS, Yueh JH, Ogunleye A, Haewyon C, Tobias AM, Lee BT. Impact of complications on patient satisfaction in breast reconstruction.PlastReconstr Surg. 2011 Apr;127 (4):1428-36. 